\title{
Ultrafast Multiphoton Thermionic Photoemission from Graphite
}

\author{
Shijing Tan, Adam Argondizzo, Cong Wang, Xuefeng Cui, and Hrvoje Petek* \\ Department of Physics and Astronomy, and Pittsburgh Quantum Institute, \\ University of Pittsburgh, Pittsburgh, Pennsylvania 15260, USA \\ (Received 5 July 2016; published 17 January 2017)
}

\begin{abstract}
Electronic heating of cold crystal lattices in nonlinear multiphoton excitation can transiently alter their physical and chemical properties. In metals where free electron densities are high and the relative fraction of photoexcited hot electrons is low, the effects are small, but in semimetals, where the free electron densities are low and the photoexcited densities can overwhelm them, the intense femtosecond laser excitation can induce profound changes. In semimetal graphite and its derivatives, strong optical absorption, weak screening of the Coulomb potential, and high cohesive energy enable extreme hot electron generation and thermalization to be realized under femtosecond laser excitation. We investigate the nonlinear interactions within a hot electron gas in graphite through multiphoton-induced thermionic emission. Unlike the conventional photoelectric effect, within about $25 \mathrm{fs}$, the memory of the excitation process, where resonant dipole transitions absorb up to eight quanta of light, is erased to produce statistical Boltzmann electron distributions with temperatures exceeding $5000 \mathrm{~K}$; this ultrafast electronic heating causes thermionic emission to occur from the interlayer band of graphite. The nearly instantaneous thermalization of the photoexcited carriers through Coulomb scattering to extreme electronic temperatures characterized by separate electron and hole chemical potentials can enhance hot electron surface femtochemistry, photovoltaic energy conversion, and incandescence, and drive graphite-to-diamond electronic phase transition.
\end{abstract}

DOI: 10.1103/PhysRevX.7.011004

\section{INTRODUCTION}

Hot carriers in graphitic materials hold enduring interest in culture, science, and technology [1-8]. In embers radiating blackbody radiation, hot carriers have provided a source of light and heat since the preindustrial age. As electricity emerged from the laboratory, the first optoelectronic devices, incandescent light bulbs, converted electricity to light by Ohmic heating of carriers within graphitized bamboo fibers. More recently, graphene has been identified as a material with remarkable optical and electronic properties for numerous applications [9-11] and has sparked interest in novel physical properties of two-dimensional (2D) materials $[12,13]$. As a semimetal, under linear optical excitation, graphene is nonemissive, but under nonlinear optical or electrical stimulation, it can emit both intense incandescence characterized by blackbody radiation temperatures in excess of $3000 \mathrm{~K}$ and broadband coherent radiation [14-17]. Moreover, strong modulation of the carrier density through

\footnotetext{
* Corresponding author. petek@pitt.edu

Published by the American Physical Society under the terms of the Creative Commons Attribution 4.0 International license. Further distribution of this work must maintain attribution to the author(s) and the published article's title, journal citation, and DOI.
}

Subject Areas: Chemical Physics,

Condensed Matter Physics, Graphene ultrafast optical excitation and the ensuing hot carrier multiplication drives the electron and hole distributions to different chemical potentials, enabling applications in energy harvesting, ultrafast electronics, and coherent optics [1,3, 16-20]. These novel properties derive from graphene's Dirac fermion band structure, weak screening, and strong, moleculelike electron correlation [21-31], which distinguish it from conventional metals and semiconductors $[22,32,33]$.

Ineffective screening of the Coulomb potential in graphitic materials favors strong carrier-carrier interactions [30]. The effectiveness of screening in a metal depends on the carrier density at the Fermi level [22]. Therefore, the minimum in the density of states (DOS) of graphene and graphite at the Fermi level makes the screening weak and prone to modification by carrier doping through photonic, electrical, or chemical stimulation $[29,34]$. Another feature owing to the 2D character of graphene and graphite is that Coulomb interaction with small momentum transfers is unscreened $[25,35,36]$. When photoexcitation and subsequent carrier multiplication suddenly raise the free carrier density above the unperturbed value, a new regime of screening with three-dimensional (3D) character can come about in graphite on a time scale of the inverse frequency of the photoexcited plasma [37,38].

Hot electron dynamics in graphene and graphite have been investigated by optical, THz, photoemission, and theoretical methods [1-9,23,34,39-46]. Xu et al. [39] first applied 
ultrafast time-resolved two-photon photoemission (TR-2PP) spectroscopy to excite a hot electron population and monitor its energy-dependent decay in graphite. Deviations of hot electron lifetimes from simple predictions of the Fermi liquid theory stimulated interest in the novel electron scattering properties of 2D materials $[39,47,48]$. Subsequent TR-2PP experiments and theoretical calculations found that the anisotropic band structure of graphite significantly constrains the phase space for electron-electron $(e-e)$ scattering $[23,41]$. Another focus has been the image potential states (IPSs) of graphite, which are the only band-structure features at the $\Gamma$ point to appear in $2 \mathrm{PP}$ spectra [Fig. 1(a)], but the mechanism for their excitation remains controversial because apposite initial states for vertical excitation do not exist $[40,42,49-51]$. Also at the $\Gamma$ point, there is a $\sigma$-symmetry interlayer band (ILB) with uniquely 3D free-electron character and band minimum at about $4.0 \mathrm{eV}$ above the Fermi level $\left(E_{F}\right.$; all electron energy levels are reported with respect to $E_{F}$ unless otherwise stated). The ILB derives its 3D character from its physical origin in hybridization of IPSs of stacked graphene sheets, which give it characteristic probability density maxima in the interlayer space. Surprisingly, the ILB has never been reported in 2PP or optical spectroscopy of graphite; however, its existence is firmly established by theory as well as inverse photoemission and x-ray absorption spectroscopies [52-57].
Ultrafast spectroscopic measurements have established several regimes of hot electron dynamics in graphene and graphite. The primary excitation between the $\pi$ and $\pi^{*}$ bands occurs with a nearly flat absorption from $1.4-3.5 \mathrm{eV}$, and thereafter increases by about $20 \%$ at $4.5 \mathrm{eV}$ because of excitonic enhancement $[9,58]$. This transition creates the primary nonthermal carrier population with electrons and holes distributed within circular cross sections through the Dirac cones at energies where photons excite vertical transitions, such as shown in Figs. 1(a) and 1(b) [9]. The primary distributions are anisotropic in momentum with the largest transition moments orthogonal to the $k$ vector of the excitation light $[59,60]$.

Immediately following the excitation, ineffective screening causes the primary distribution to undergo rapid energy and momentum redistribution through $e-e$ and $e-p$ scattering $[21,25,61,62]$. Electron energy relaxation occurs through Coulomb interaction where two electrons scatter into two unoccupied hole states in a process that conserves both energy and momentum [22,63-65]. Note that $e-e$ scattering is strongly excitation fluence and time dependent because of evolving electron and hole distributions. Moreover, kinematic constraints localize the scattering primarily within the Dirac cones (intraband) rather than between them (interband) [65].

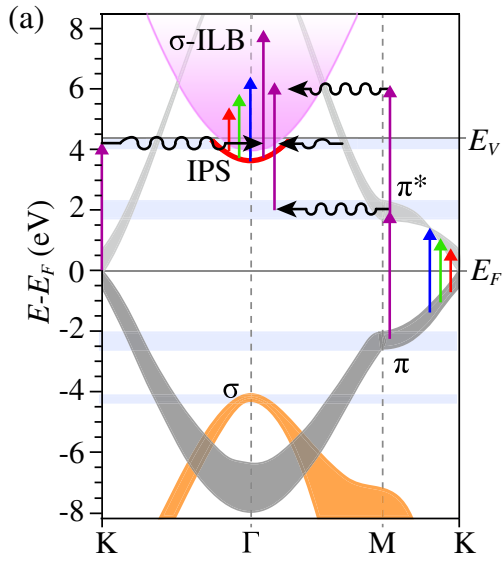

(b)

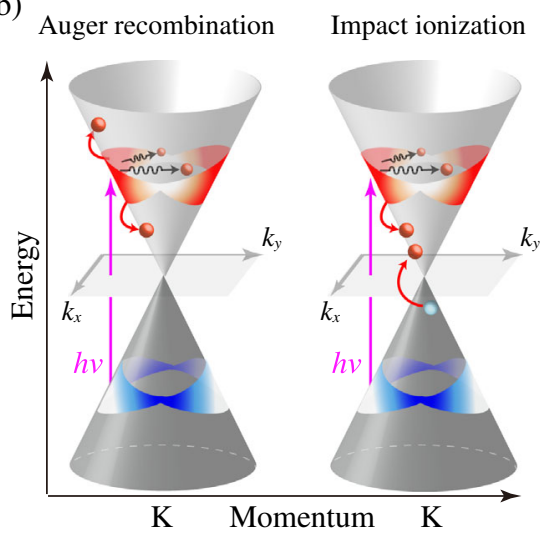

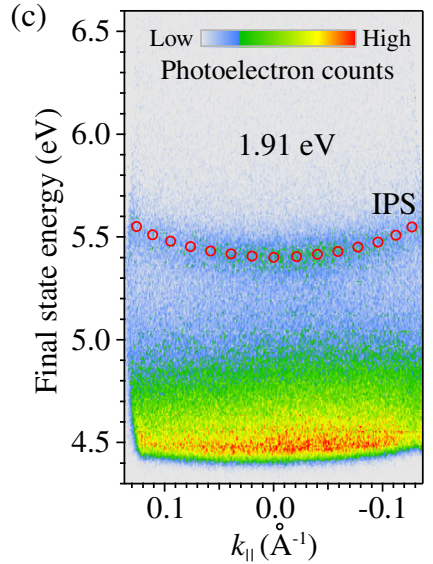

FIG. 1. (a) The $k_{\|}$band structure of graphite in the $\Gamma-\mathrm{M}-\mathrm{K}$ direction. The bandwidths represent the perpendicular band dispersions. Resonant optical absorption from IR to UV in graphite occurs by vertical dipole transitions between the $\pi$ and $\pi^{*}$ bands. Light blue shaded rectangles denote regions of high densities of states (van Hove singularities), where intervalley momentum scattering among the $\sigma$ and $\pi$ bands is particularly strong. Hot electron thermalization populates the image potential state (IPS; the red curve) and the 3D interlayer $\sigma$ band (ILB; pink shaded area); the former is detected by one-photon photoemission, and the latter is the source of thermionic emission. Electron-phonon scattering (wavy arrows) mediates indirect $2 \mathrm{PP}$, which is initiated by $\pi$ - $\pi^{*}$ excitation, and it also transfers hot electron population among the $\sigma$ and $\pi$ bands. (b) The primary photoexcited density is distributed anisotropically in circular cross sections through the Dirac cones at energies that are connected by the $\pi$ - $\pi^{*}$ transition with the maximum density perpendicular to the propagation vector of the excitation light $\left(k_{x}\right)$. Red above and blue below $E_{F}$ denote the primary hot electrons and hot holes; dark grey represents the occupied states prior to excitation. The primary distribution is thermalized within 25 fs by $e$ - $e$ scattering processes; Auger recombination causes carrier heating, whereas carrier multiplication by impact ionization is suppressed by limited phase space for the collinear process. Simultaneously, momentum is randomized by $e-p$ scattering (wavy arrows). (c) A typical $E(k) \mathrm{mPP}$ spectral distribution plotting the final photoelectron energy $E$ vs parallel momentum $k$ of graphite for $p$-polarized $h \nu=1.91 \mathrm{eV}$ excitation. The dispersive IPS (indicated by red circles) is superimposed on a TES background, whose counts decay exponentially above the work-function edge at $4.4 \mathrm{eV}$. 
In Fig. 1(b), we describe the scattering processes of the primary photoexcited carriers, which are localized in energy and momentum via the electric-dipole excitation in graphite; our discussion assumes that the photoexcited density significantly exceeds that at the Fermi level. In the case of impact ionization, a primary hot electron scatters with an electron in the Fermi sea below $E_{F}$ to produce two lower-energy hot electrons; this multiplies the number of hot electrons, while reducing their energy, and equilibrates the conduction and valence bands. Because this is an interband process, it occurs with a low probability $[4,45,65]$. By contrast, Auger recombination involves the scattering of two hot electrons, where one gains and the other one loses the same amount of energy [4,5,8]. Using 7 fs near-IR laser pulses, Breusing et al. $[1,8]$ found that, in graphene and graphite, such intraband scattering with a large phase space thermalizes the carriers to separate electron and hole chemical potentials within 30 fs $[1,8,45,65]$. The conduction electrons undergoing multiple intraband $e-e$ scattering interactions can thus gain several $\mathrm{eV}$ energy [66]. The screening and kinematic constraints thus make $e$-e scattering in graphitic materials much faster and more effective in reaching high temperatures than is possible in semiconductors or metals $[33,67]$. In other words, because of the ineffective screening and smaller heat capacity in graphitic materials, as compared with metals, thermalization happens on much shorter time scales before photoexcited electrons can dissipate a sizable amount of energy to the lattice. Thus, for the same amount of absorbed energy, electronic temperatures in photoexcited graphite will be much higher than in photoexcited metals.

In parallel with $e-e$ scattering, $e-p$ scattering makes the carrier distributions more isotropic [46]. Although momentum transfers in $e-p$ scattering can be large, the energy transfers are limited by the frequency of optical phonons $[6,34,45]$. Therefore, $e-p$ scattering has a much smaller effect on the energy relaxation of the primary hot electron distribution. Once the carrier Fermi-Dirac distributions are established, energy transfer by the $e-p$ scattering to the lattice occurs on $\sim 1$-ps time scale [3,5].

Here, we investigate the hot electron generation and thermalization in graphite by ultrafast time-resolved multiphoton photoemission (TR-mPP) spectroscopy using broadly tunable, IR-UV excitation. The photoelectron yields are remarkably nonlinear with the laser fluence $I$, having power-law dependence $I^{m}$, where $m$ ranges from 2 to 8 as the excitation wavelength is tuned from UV to IR. High-order nonlinear mPP processes have been observed in metals in the perturbative and nonperturbative regimes of light-matter interaction, where electrons are promoted from occupied bands in an energy ladder-climbing process by absorbing multiple $m$ quanta of light, $h \nu$, or are accelerated and released into the vacuum by the applied optical field [68-70]. By contrast, mPP in graphite occurs in an entirely different regime, where absorption of $m$ quanta of light by the $\pi-\pi^{*}$ transition creates the primary hot electrons. The primary hot electrons, however, do not have an upward resonant photoexcitation pathway, but rather, the nonlinearity in photoemission arises through Auger heating among hot electrons, which enables them to gain several $\mathrm{eV}$ energy within a thermalization time of less than $25 \mathrm{fs}$ to achieve statistical Boltzmann distributions with electronic temperatures up to $T_{e}=5,500 \mathrm{~K}$. This thermalized population is sufficiently hot to partially populate the ILB above the vacuum level, $E_{v}=4.4 \mathrm{eV}[52,54,71,72]$, from where electrons undergo thermionic emission. The ultrafast scattering processes that lead to thermionic emission obliterate information in $\mathrm{mPP}$ spectra on the electronic bands and dipole transitions through which the primary excitation occurred. The nearly instantaneous augmentation of the free carrier density above about $10^{19} \mathrm{~cm}^{-3}$ at the Fermi level alters the chemical and physical properties of graphite [34,73-76]. In particular, we propose that the reported transformation of $\mathrm{sp}^{2}$ graphite to $\mathrm{sp}^{3}$ diamond under femtosecond laser excitation [34,74-77] occurs by a photoinduced electronic phase transition: The redistribution of electrons from the $\pi$ bands with bonding character to the unoccupied ILB causes the lattice instability.

\section{EXPERIMENTAL METHODS}

TR-mPP measurements [38,78] are performed on a highly oriented pyrolytic graphite (HOPG). The sample surface is cleaved in air and degassed at $900 \mathrm{~K}$ for 10 hours in the ultrahigh vacuum chamber (base pressure $<1 \times 10^{-10} \mathrm{mbar}$ ) for photoemission measurements. All measurements are performed at room temperature; cooling the sample to about $100 \mathrm{~K}$ has no discernible effect on $\mathrm{mPP}$ spectra.

$\mathrm{mPP}$ from graphite is excited with a tunable ultrafast laser spanning the 880-290 $\mathrm{nm}(h \nu=1.41-4.28 \mathrm{eV})$ spectral range. Excitation pulses are generated by a noncollinear parametric amplifier (NOPA), which is pumped by a Clark MXR Impulse fiber-laser oscillator amplifier. Using the second or third harmonic of the fiber laser for pumping and the fundamental for white-light generation, the NOPA produces light in the 660-900-nm (1.88$1.38-\mathrm{eV})$ and $480-660-\mathrm{nm}(2.58-1.88-\mathrm{eV})$ bands, respectively, with typically about $20-30$-fs pulse duration. The NOPA outputs are compressed by multiple reflections from matched pairs of negative dispersion mirrors to produce the shortest autocorrelation traces at the sample position. Frequency doubling of the NOPA output in BBO crystals produces UV light in the 270-450-nm (4.59-2.76-eV) band. At a $1.25-\mathrm{MHz}$ pulse repetition rate, the average excitation powers are in the $10-80-\mathrm{mW}$ range for the fundamental IR-VIS output and $0.1-10 \mathrm{~mW}$ for the frequency-doubled UV output. $p$ - or $s$-polarized excitation is selected with $\lambda / 2$ plates. The NOPA output beam is focused onto the sample in the photoemission chamber at $45^{\circ}$ from the surface normal. A bias of $2 \mathrm{~V}$ is applied between the HOPG sample and the analyzer to collect 
near-zero kinetic energy electrons. The photoemitted electron energy $E$ vs parallel momentum $k_{\|}$distributions, $E(k)$, are analyzed and recorded with a Specs Phoibos 100 hemispherical analyzer with a 2D delay line detector. The analyzer acceptance angle is $\pm 15^{\circ}$ [78].

A Mach-Zehnder interferometer generates identical pump and probe pulses and scans their delay to record interferometric two-pulse correlations (I2PCs) of hot electron dynamics $[79,80]$. At each time delay increment of about 50 as, an $E(k)$ distribution is acquired as described elsewhere [38]. To measure delay-dependent hot electron temperatures, the pump-probe delay is fixed to specific values corresponding to integral numbers of optical cycles, and $E(k)$ photoelectron distributions are acquired for the double pulse excitation.

\section{RESULTS}

mPP spectroscopy usually measures the energy levels and band dispersions of the initial, intermediate, and final states that are coupled by optical transitions in a multiphoton absorption process [81-83]. According to the band structure of graphite in Fig. 1(a), there is a gap of about $7.4 \mathrm{eV}$ from the highest occupied bulk $\sigma$ band [84] to the lowest unoccupied IPS, which makes the direct excitation at the $\Gamma$ point $\left(k_{\|}=0 \AA^{-1}\right)$ unlikely. Therefore, $\mathrm{mPP}$ spectra of graphite at the $\Gamma$ point must involve energy and momentum scattering of photoexcited carriers. In the following, we use the wavelength, polarization, and power dependence of mPP spectra to extract information on the band-structure effects and scattering processes that define them.

Figure 1(c) presents an mPP spectral image of the finalstate photoelectron energy $E$ vs parallel momentum $k_{\|}$ resulting from multiphoton excitation with $p$-polarized $h \nu=1.91 \mathrm{eV}$ light. The spectrum combines a dispersive band with a minimum at the $\Gamma$ point, which is superimposed over a featureless background signal, whose intensity decays exponentially above $E_{v}$. These spectral components will be identified shortly.

To gain an overview of photoinduced dynamics in graphite, in Fig. 2(a), we present mPP spectra normalized at the work-function edge for the entire excitation $h \nu$ range, where each trace is a $k_{\|}=0 \AA^{-1}$ cross section through a 2D spectrum, such as in Fig. 1(c). We identify three distinct contributions to the mPP spectra: First, there is a sharp peak, whose intensity is relatively strong for IR-VIS excitation up to $h \nu=2.6 \mathrm{eV}$, where it drops off and rises again in the UV for $h \nu \geq 3.6 \mathrm{eV}$. This peak comes from the dispersive band in Fig. 1(c). By plotting how the final-state energy $E$ of this peak shifts with $h \nu$ [Fig. 2(b)], we can determine, from a slope of about 1 and an intercept of $3.6 \mathrm{eV}$, that this is the $n=1 \mathrm{IPS}$ of graphite. How the IPS is populated is yet unclear, but it is detected by absorbing one additional photon; hence, $E=E_{I P}+h \nu$. The IPS energy $E_{I P}=3.6 \mathrm{eV}$ with respect to $E_{F}$ and the corresponding binding energy of $0.8 \mathrm{eV}$ with respect to $E_{v}$ conform with the previous reports for graphite $[40,42,49,50]$. According to IPS wave-function symmetry, only the surface-normal
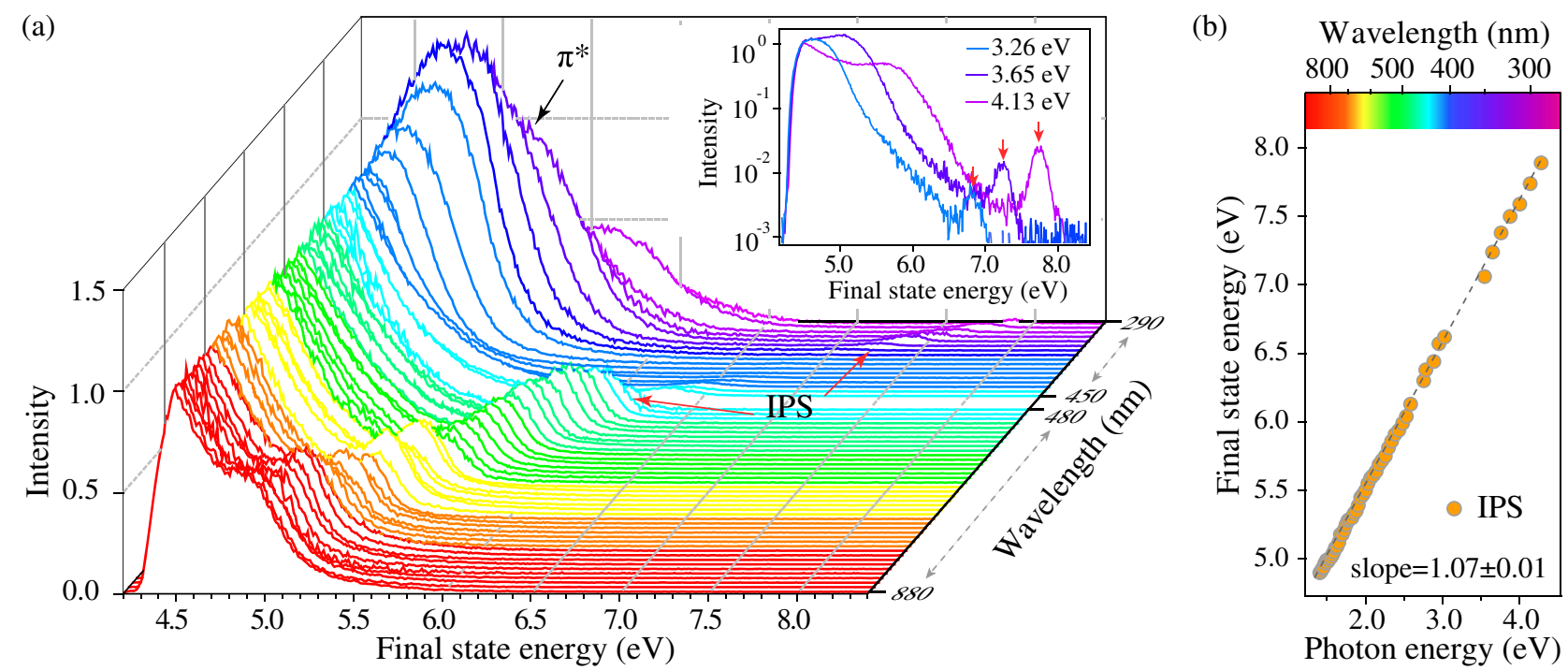

FIG. 2. (a) A series of mPP spectra of graphite for $p$-polarized excitation taken in 10 -nm intervals from 880 to $290 \mathrm{~nm}$ ( $h \nu=1.41-4.28 \mathrm{eV}$; there is a tuning gap between 480 and $450 \mathrm{~nm}$ ), which are obtained by taking $k_{\|}=0$ cross sections through $E(k)$ distributions such as in Fig. 1(c). The spectra are normalized at the work-function maximum (4.5 eV). Red arrows point to the IPS peak. The inset shows several spectra with UV excitation on a log-vertical scale to emphasize the weak signal from IPS. Above $h \nu=2.95 \mathrm{eV}$, there is an additional component due to the indirect excitation from the $\pi$ band. (b) A plot of the IPS final-state energy vs the photon energy, $h \nu$. The slope (1.07) and intercept (3.6 eV) identify the IPS as the penultimate state in $\mathrm{mPP}$ at $0.8 \mathrm{eV}$ below $E_{v}$ or $3.6 \mathrm{eV}$ above $E_{F}$. 
component of $p$-polarized light can excite it into the photoemission continuum [32]. The continuous mPP background signal, however, appears for both $s$ and $p$ polarizations; therefore, subtracting normalized spectra separates IPS from the background signal [Fig. 3(a)].

The second component in the mPP spectra, which becomes evident for $h \nu>2.95 \mathrm{eV}$, can also be discerned by subtracting the normalized $s$ - and $p$-polarized spectra [Fig. 3(b)] because its transition moment is relatively larger for $p$ polarization. It has been assigned to a 2PP process that is initiated by resonant excitation from the initial $\pi$ to intermediate $\pi^{*}$ bands [Fig. 1(a)] [40,42]. Further excitation of the primary hot electron in the $\pi^{*}$ band is unlikely because there are no vertical bands above it within about $6 \mathrm{eV}$, where such transitions could terminate. Moreover, to be photoemitted, electrons must have sufficient kinetic energy in the surface-normal direction to overcome the surface potential [68]. Hot electrons near the $\mathrm{K}$ and $\mathrm{M}$ points, however, have most of their kinetic energy in the surface parallel motion and therefore cannot undergo photoemission after absorbing another photon, unless they also undergo momentum scattering. Therefore, this $\pi^{*}$ band component can only be photoemitted by a second-order process involving photon absorption and concomitant momentum scattering into the $\Gamma$ valley, as indicated in Fig. 1(a) [85].

The third component of the mPP spectra, which is responsible for most of the photoemission signal, is the primary focus of our study. It is the background signal with exponentially waning counts above the work-function edge that dominates the mPP spectra in Fig. 2(a). For IR-VIS excitation, it is the only contribution to the $s$-polarized spectra, as is evident in Fig. 3(a). This is the thermionic emission signal (TES) from the hot electron gas that is produced through the multiphoton absorption and subsequent electron thermalization; it is a direct measure of the hot electron distribution above $E_{v}$.

To further characterize the TES and IPS contributions to the $\mathrm{mPP}$ process in graphite, in Figs. 3(c) and 3(d) we report the power-law dependence $I^{m}$ of their mPP yields on the laser fluence for $h \nu=3.88$ and $1.70 \mathrm{eV}$. The slopes of logarithmic plots in Figs. 3(c) and 3(d) give the power-law exponent $m$ for the mPP yield. Figure 3(e) reports how $m$ varies with $h \nu$. In the UV region, the energy of two photons is sufficient to overcome the work function and therefore induce photoemission. We find threshold energies for
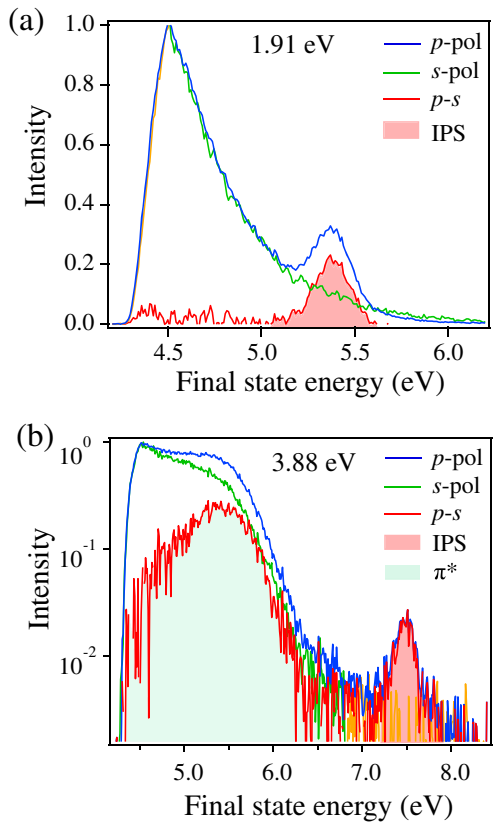

(c)

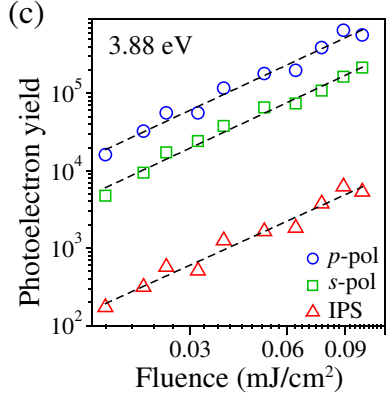

(d)

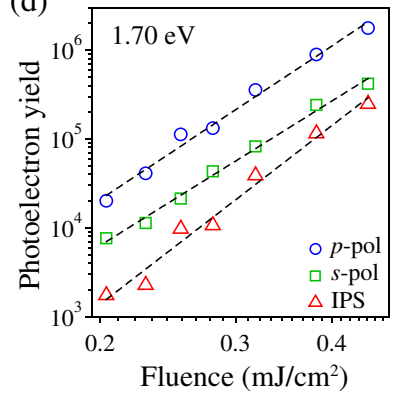

(e)

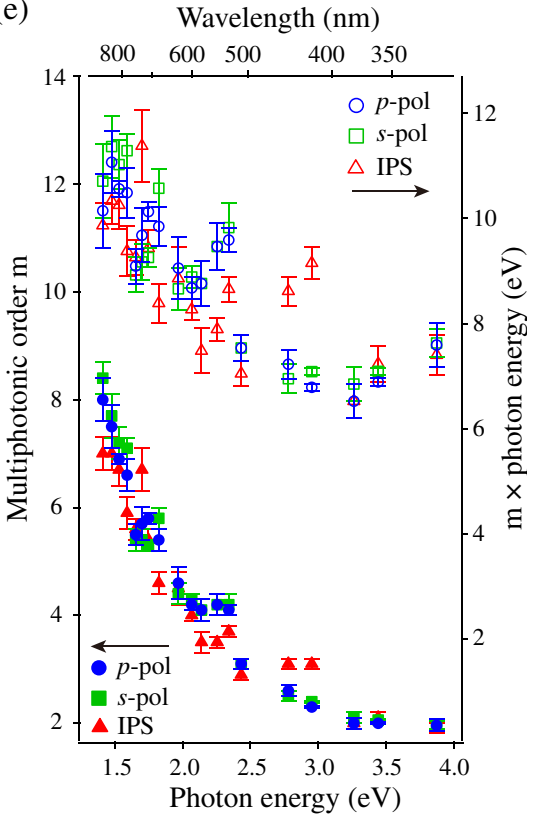

FIG. 3. Identification of different spectral components contributing to mPP spectra and their nonlinear order. (a) Normalized mPP spectra obtained with $p$ polarization (blue) and $s$ polarization (green) for $h \nu=1.91 \mathrm{eV}$. The difference spectrum between the $p$ - and $s$-polarized spectra (red curve) isolates the IPS (pink). (b) Normalized mPP spectra obtained with $p$-polarized (blue) and $s$-polarized (green) light for $h \nu=3.88 \mathrm{eV}$ and their difference representing photoemission from the $\pi^{*}$ state (aquamarine) and IPS (pink). (c) The photoelectron yields with increasing laser fluence obtained by integrating the photoelectron counts for $p$-polarized (blue) and $s$-polarized (green) excitation. For IPS, the photoelectron yield is integrated from the pink area in the difference spectra such as in (a). The $\log$ - $\log$ plots give slopes of 1.96, 1.97, and 1.91 for the $\mathrm{p}, \mathrm{s}$, and IPS spectra, respectively, corresponding to the nonlinear order of $m \sim 2$. (d) The same plot as in (c) for $h \nu=1.70 \mathrm{eV}$; the obtained slopes are 5.74, 5.40, and 6.68 for the p, s, and IPS spectra, respectively, corresponding to $m \sim 6$. (e) Left axis: The multiphotonic order $m$ for the $p$ - and $s$-polarized spectra, as well as the IPS component vs $h \nu$ (solid symbols). Right axis: The total photon energy absorbed $(m h \nu)$ that is required to photoemit an electron from graphite vs $h \nu$ (hollow symbols). 
which $m=2$ of $h \nu>3 \mathrm{eV}$ for the TES and $h \nu>3.6 \mathrm{eV}$ for the IPS [40,42]. As $h \nu$ decreases, however, $m$ increases markedly up to about 8 for the lowest energy $h \nu=$ $1.41 \mathrm{eV}$. The higher exponent in the IR-VIS region cannot be attributed to variations in the optical absorption of graphite $[9,58]$. Having obtained the exponent, we can determine the average energy absorbed by graphite per photoemitted electron (the nonlinear exponent times the photon energy, $m h \nu$ ), which ranges from $8 \mathrm{eV}$ in the $\mathrm{UV}$ to $12 \mathrm{eV}$ in the IR spectral regions [Fig. 3(e)]; i.e., in the entire $h \nu$ range, it significantly exceeds the work function of graphite. A single electron never absorbs the entire energy of $m$ photons because TES extends only $1-2 \mathrm{eV}$ above $E_{v}$. This is contrary to perturbative mPP spectra of metals, where single electrons absorb $m$ photons from the occupied bands so the maximum photoelectron energy appears at $E_{F}+m h \nu$. Moreover, if multiple orders of $m$ appear in $\mathrm{mPP}$ spectra, the photoelectron yield strongly decreases with each higher order $[68,69]$.

The increase of $m$ with decreasing $h \nu$ can be understood in the following way. In the case of photoexcitation with, for example, $h \nu=1.5 \mathrm{eV}$, the electron and hole, on average, receive $0.75 \mathrm{eV}$ energy. Because the photoexcited electron density is significantly larger than at $E_{F}$ and intraband scattering dominates over interband scattering, the $e$-e scattering will occur dominantly among the primary hot electron population. Thus, the maximum energy that an electron can gain in each scattering event among this hot population will be $0.75 \mathrm{eV}$, and therefore, at least five scattering events will be necessary to excite a small fraction of the electrons above $E_{v}=4.5 \mathrm{eV}$, where they can be emitted as TES. Naturally, if $h \nu$ is larger, the number of required scattering processes will be lower and therefore $m$ will be smaller.

Next, we quantify how the TES energy distributions depend on the photon energy and laser fluence. Thermionic emission corresponds to hot thermalized electrons excited by $e$-e scattering to energies above $E_{v}$ escaping into the vacuum. Thus, we expect TES to conform to a statistical Boltzmann energy distribution of a nondegenerate hot electron gas: $I(E) \propto \exp \left[-E /\left(k_{B} T_{e}\right)\right]$, where $k_{B}$ is the Boltzmann constant $[14,15,28]$. The density of states of graphite for threshold photoemission is flat [53] and therefore not a factor in our analysis. Similar assumptions of thermal equilibrium for the electronic system were made to analyze the ultrafast multiphoton excitation of blackbody luminescence from graphene, thermionic electron emission from carbon nanotube forests, and electron or ion emission from $\mathrm{C}_{60}[14,15,28,86,87]$. Indeed, fitting representative $s$-polarized mPP spectra for $h \nu=1.48 \mathrm{eV}$ obtained with different fluences gives the $I$-dependent values of $T_{e}$ [Figs. 4(a) and 4(b)]. Measurements of TES at other wavelengths show that smaller $h \nu$ corresponding to higher values of $m$ give hotter $T_{e}$ [Fig. 4(b)], which is consistent with the trend in energy absorbed per photoelectron, $m h \nu$,
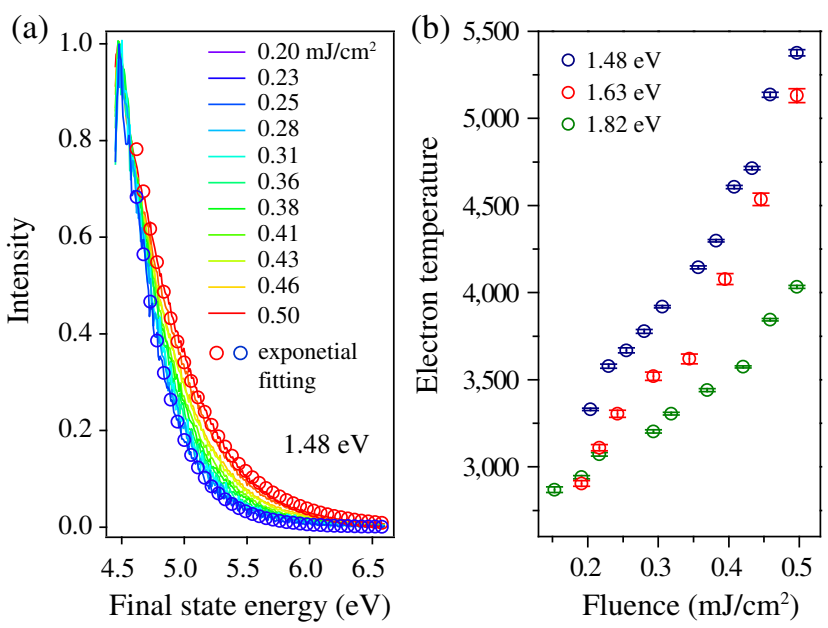

FIG. 4. (a) Exponential fitting of the normalized s-polarized $\mathrm{mPP}$ spectra for different laser fluences and $h \nu=1.48 \mathrm{eV}$. (b) The fluence-dependent temperatures for $s$-polarized $\mathrm{mPP}$ spectra excited with $h \nu=1.48,1.63$, and $1.82 \mathrm{eV}$. Note that light quanta $h \nu$ with smaller energy produce hotter electron distributions.

in Fig. 3(e). Moreover, the high-order $m$ for the IR excitation makes $T_{e}$ extremely sensitive to the laser fluence: Doubling $I$ can increase $T_{e}$ by about $2000 \mathrm{~K}$, with $5500 \mathrm{~K}$ being achieved for only $I \sim 0.5 \mathrm{~mJ} / \mathrm{cm}^{2}$. Thus, the fluence-dependent Boltzmann photoelectron energy distributions confirm that TES is thermionic emission that is produced by thermalization of the hot electron plasma.

To get more insight into the electron dynamics that produce the thermionic emission, we measure time-domain interferograms of mPP [38]. Changing the delay $\tau$ between identical collinear pump and probe pulses in 50-as steps while recording $E(k)$ distributions of $\mathrm{mPP}$ records interferometric movies of the nonlinear TES excitation. Figure 5(a) shows an interferogram for excitation by $h \nu=1.97 \mathrm{eV}$, which represents a cut through such a movie for $k_{\|}=0 \AA^{-1}$ [38]. Vertical and horizontal cross sections through this interferogram give, respectively, the mPP spectrum for $\tau=0$ fs [Fig. 5(a)] and a I2PC scan for $E=4.6 \mathrm{eV}$ [Fig. 5(b)]. The I2PC scan oscillates coherently with the period of the optical carrier wave (2.10 fs), which is caused by the coherent interaction between the pump and probe pulses during their temporal overlap of $\pm 30 \mathrm{fs}$. This interference signal is not expected to give information on dephasing of coherent polarization fields in the sample because the inhomogeneous broadening of the $\pi-\pi^{*}$ transition of graphite is much larger than the laser bandwidth; therefore, its coherence time is shorter than that of the excitation field. In mPP from metals, such interferometric experiments measure the coherent response of the sample at the driven or induced frequencies as well as the population dynamics in real intermediate states [38,79]. In the case of thermionic emission, however, the I2PCs 

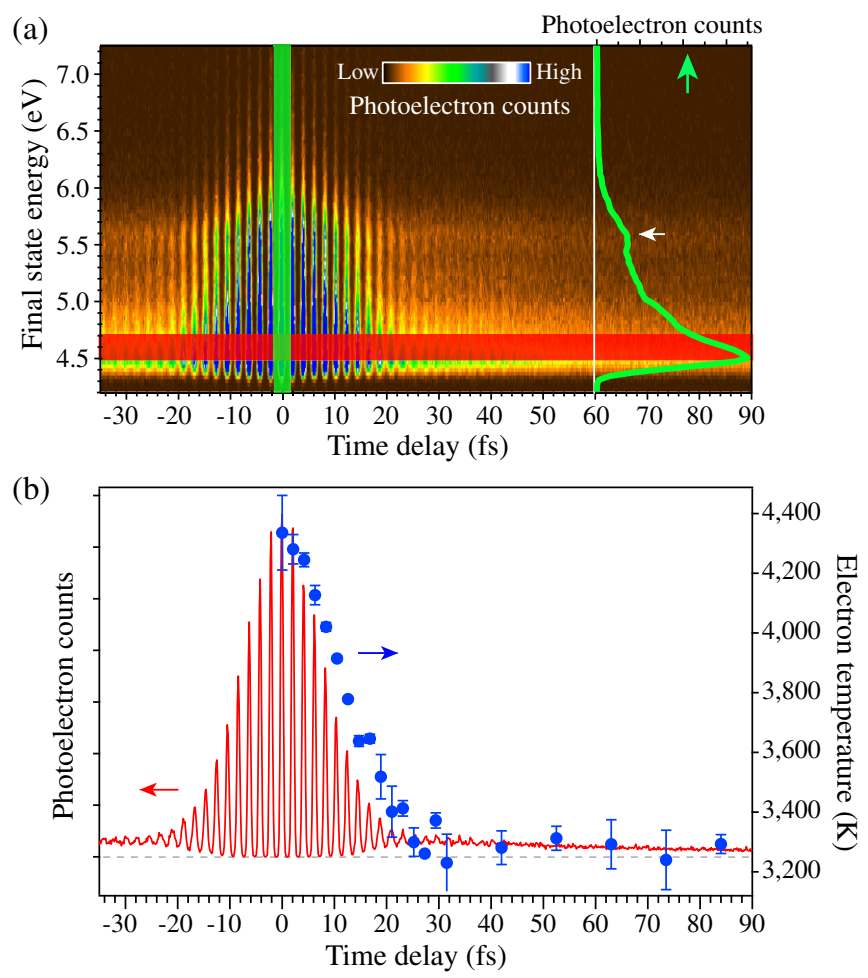

FIG. 5. Interferometric two-pulse correlation (I2PC) measurements of mPP. (a) $k_{\|}=0 \AA^{-1}$ cut through a 3D movie of mPP intensity vs energy, momentum, and delay time $\tau$, for excitation with $p$-polarized $h \nu=1.97 \mathrm{eV}$ light. The integration of the mPP signal within the green shaded rectangle over $\tau$ gives the $\mathrm{mPP}$ spectrum at $\tau=0$ (green line; the green arrow points to the intensity scale). The white arrow points to the IPS peak. (b) Integration of the $\mathrm{mPP}$ signal within the red shaded rectangle in panel (a) over $E$ gives the I2PC trace at $E=4.6 \mathrm{eV}$ (red). The dashed line is the zero line for the I2PC scan. The blue dots give the hot electron temperature following the two-pulse excitation with $s$-polarized light for selected delays corresponding to an integral number of optical cycles.

measure the final-state energy distribution above $E_{v}$ integrated over the cooling time, rather than dephasing or scattering processes in intermediate states of the mPP process.

Because of the thermionic character of photoemission, it is inappropriate to interpret the I2PC data in terms of dynamics of electron populations at specific energy levels as in a conventional mPP process [88]. Instead, we extract information from the I2PC scans by determining the delay-dependent $T_{e}$ from TES recorded at the interference fringe maxima. This procedure gives the plot of $T_{e} v s \tau$ in Fig. 5(b), which shows that the double pulse excitation enhances $T_{e}$ but only within the first 25 fs. For $\tau>25$ fs, the two pulses heat the electron gas independently without an enhancement by a correlated nonlinear interaction, and $T_{e}$ plateaus at a constant value more than $1000 \mathrm{~K}$ below that at $\tau=0$. This is surprising because after $25 \mathrm{fs}$, the energy deposited by the pump pulse still remains predominantly in the electronic system, but the electronic properties of graphite, namely, screening, have changed such that the nonlinear mechanism for the correlated heating of the electron gas by both pulses has elapsed. Based on these observations, we consider the origin of the ultrafast scattering processes that give rise to TES in graphite.

\section{RESULTS AND DISCUSSION}

The TR-mPP measurements reveal a remarkably nonlinear response of graphite to optical excitation, which creates a hot thermal photoelectron distribution on time scales less than 25 fs. In ultrafast photoelectron imaging experiments using 10-100 times higher fluences, thermionic photoemission from graphite with $T_{e}$ that saturates at $18,000 \mathrm{~K}$ has already been deduced, but its origin has not been discussed [89]. In light of typical mPP spectra of metals, where dipole transitions rather than energy and momentum scattering play the dominant role in the photoemission process $[68,69]$, the rapid thermalization in graphite raises the question of the mechanism for the nonlinear electron excitation in graphite.

Figures 1(a) and 1(b) illustrate the possible electron excitation pathways by photon absorption and $e$-e scattering in graphite. According to the characteristic band structure of graphite and graphene, dipole transitions for the entire $h \nu$ range of our experiments occur exclusively between the $\pi$ and $\pi^{*}$ bands forming the Dirac cones [9,58]. Because $E_{F}$ is near the Dirac point, the photon energy is nearly equally split between the hot electron and the hot hole. Once an electron is promoted to the $\pi^{*}$ band, there is no vertical excitation pathway. Thus, populating unoccupied bands near $k_{\|} \sim 0 \AA^{-1}$, namely, the ILB and IPS, requires the primary hot electrons to gain several $\mathrm{eV}$ of energy. This can only happen by Auger heating through multiple scattering events.

Three factors can promote carrier excitation through scattering processes: First, the Dirac fermion band structure supports strong resonant $\pi-\pi^{*}$ excitation [Figs. 1(a) and 1(b)] throughout the IR to UV regions $[9,58]$. This linear absorption process creates a hot nonthermal electron gas at approximately $1 / 2 h \nu$ energy above $E_{F}$. The transition can saturate because of the Pauli blocking, unless out-scattering rapidly empties the photoexcited states. Second, $e-e$ scattering processes that thermalize the electronic system within about 25 fs $[1,4,21]$ counteract the Pauli blocking. In particular, Auger recombination among hot electrons heats a fraction of them to energies above $E_{v}$. The order $m$ of the nonlinear process is large when $h \nu$ is small because more scattering events among the hot primary population are necessary to heat the electrons above $E_{v}$ than when $h \nu$ is large. Third, intervalley momentum scattering through the $e-p$ deformation potential interaction, which is pronounced at van Hove singularities [85], such as at the bottom of the ILB and the M 
point of the $\pi^{*}$ band, transfers hot electrons among the $\pi^{*}$, $\sigma$, and image potential bands. These energy and momentum scattering processes within the hot electron gas populate the IPS and ILB of graphite [52,54] and therefore give rise to the mPP signal at the $\Gamma$ point.

Thus, the nonlinearity in the $\mathrm{mPP}$ process must arise from $e-e$ scattering in the hot electron gas in the photoexcited phase space rather than from multiple-photon absorption by single electrons. In this scenario, the exponent of the mPP process $m$ must increase as $h \nu$ decreases because the primary hot electrons receive less energy, and therefore, for them to be excited above $E_{v}$, they must suffer more Auger interactions that transfer less energy than for hot electrons that are generated with larger $h \nu$. We also note that the Auger scattering rate has a quadratic dependence on the hot electron density [66]. We estimate that the primary hot electron density under our experimental conditions is in the $10^{20} \mathrm{~cm}^{-3}$ range, i.e., an order-ofmagnitude higher than the density at $E_{F}$ of unexcited graphite [35]. This primary population is larger than the screening charge density at the Fermi level and thus is subject to strong Coulomb interactions until carrier correlations are established on the time scale of the photoexcited plasma frequency $[4,37]$. Thus, the cascaded Auger heating of the electron gas through ineffectively screened Coulomb interactions with nonlinear density dependence can explain the observed high-order power-law behavior of TES.

The IPS signal intensity in Fig. 2(a) shows strong dependence on $h \nu$ and thus is an indicator of excitation and scattering processes that lead to its population. The excitation of the IPS with $3.1 \mathrm{eV}$ light has been discussed in previous 2PP and 3PP studies of graphite. Takahashi et al. proposed that vertical excitation of IPS near the $\Gamma$ point occurs from the occupied $\sigma$ band [Fig. 1(a)] [49]; such resonant $2-5$ photon band-to-band transitions are possible in our $h \nu$ range but must be weak because we find no evidence for resonance enhancement of the $\mathrm{mPP}$ signal at the expected $E$ and $k_{\|}$. Our measurements show instead that for $h \nu<2.6 \mathrm{eV}$, the IPS signal is intense, its strength relative to TES is nearly constant, and its excitation order is $m \geq 3$. Therefore, like TES, in the IR-VIS region, the population of IPS is consistent with a statistical distribution of hot electrons in the unoccupied bands of graphite.

For the UV excitation, where the photoemission predominantly occurs by a two-photon process, the IPS contribution to $\mathrm{mPP}$ spectra is significantly weaker than the vacuum edge signal as compared with the IR-VIS excitation. The 2PP processes with UV excitation can occur by second-order phonon-induced scattering [Fig. 1(a)], as discussed already. Similar momentum scattering processes also explain why the IPS intensity starts to increase at $h \nu=3.6 \mathrm{eV}$ and reaches a local maximum for $h \nu=4 \mathrm{eV}$ [Fig. 2(a), inset] [42]. These energies are thresholds for the indirect excitation channel from $E_{F}$ to IPS and ILB via the simultaneous photon absorption and momentum scattering
[Fig. 1(a)]. The local maximum of the IPS intensity at $h \nu=4 \mathrm{eV}$ previously attributed by Pagliara et al. to a putative excitation-induced band-gap renormalization of the $\pi$ bands of graphite $[42,90]$ more likely portends the onset of the phonon-mediated excitation of ILB from the $E_{F}$.

Although the IPS can be populated through hot electron thermalization, it cannot appear in $\mathrm{mPP}$ spectra as a distinct spectroscopic feature through a thermionic process. The 2D character of IPS makes electrons populating it bound for all energies [68] because their kinetic energy is entirely in the surface parallel motion. Therefore, IPS electrons can undergo thermionic emission only by scattering into the photoemission continuum whereby their distinct energymomentum dispersion is obliterated. The only way to detect electrons in the IPS is for them to absorb another photon to undergo photoemission. Electrons populating the ILB could also be photoemitted, but an onset of this process expected at its band minimum corresponding to an intermediate-state energy of about $4 \mathrm{eV}$ is notably absent in $\mathrm{mPP}$ spectra. Most likely, the photoexcitation from ILB has a small transition moment. Instead, the ILB serves as a reservoir of hot electrons from which the thermionic emission occurs. Moreover, the population of ILB enables $3 \mathrm{D}$ screening in otherwise 2D material. The existence of ILB in graphite and lack thereof in graphene is the main difference between the two materials as far as thermionic emission is concerned.

\section{CONCLUSIONS}

We have investigated the population of IPS and ILB by ultrafast Auger heating within the photoexcited hot electron gas leading to thermionic emission from graphite surfaces. Because the IPS is a universal property of molecular sheets [56], the ILB is derived from IPS by stacking them into a 3D solid [57]; screening is inefficient, and similar hot electron dynamics could occur in other quasi-2D materials with low densities of states at the Fermi level. Moreover, thermionic emission can occur in other materials and is likely to be the dominant process for photoemission from plasmonic nanoparticles where hot electron generation and light localization are particularly efficient [91-93].

Based on our measurements of the energy and timedependent photoelectron spectra, the following scenario for the thermionic $\mathrm{mPP}$ of graphite emerges. The sparse band structure of graphite, exceptionally strong broadband absorption by the $\pi-\pi^{*}$ transition, and weak screening of the Coulomb interaction enable multiple quanta of light to be deposited into the electronic system within 25 fs. Hot nonthermal electrons and holes of this primary distribution interacting through ineffectively screened Coulomb interaction scatter throughout the available phase space, including the $\sigma$ bands, to achieve a Boltzmann distribution with temperatures as high as $5500 \mathrm{~K}$. Electrons excited by IR light must gain more than $3 \mathrm{eV}$ by Auger scattering processes within 25 fs to be photoemitted. Such extreme 
electron correlation can be attributed to ineffective screening of the Coulomb interaction and resembles electron relaxation processes in molecular materials such as $\mathrm{C}_{60}$ and carbon nanotubes rather than metals [28,87]. In metals, where the Coulomb interaction is more strongly screened and the photoexcited carrier density is only a small fraction of the Fermi-level density [38], reaching comparable $T_{e}$ requires about 100-times-larger fluences and electron thermalization takes about 10 times longer [67,73,94,95].

The excitation of electrons from the $2 \mathrm{D} \pi$ bands to the 3D $\sigma$ band can enhance processes such as hot electron chemistry and nanodiamond synthesis. Hot electrons mediate surface photochemistry on metals, but fast electronic quenching of molecule-localized states in resonance with the conduction band renders the quantum yields negligibly small [96-99]. The strong $\pi-\pi^{*}$ transition in graphite makes the hot electron excitation far more efficient than in metals, which dominantly reflect light. The ILB or the van Hove singularity regions at the $\mathrm{M}$ point in the $\pi$ bands can act as hot electron reservoirs from which charge transfer to adsorbed molecules and thus surface femtochemistry can effectively occur. Further enhancement of photosensitized processes is likely for molecular acceptor states, which lie within the wide $\sigma$ and $\pi$ gaps of graphitic surfaces, because quenching of molecule-localized resonances by the reverse charge transfer to resonant states with large $k_{\|}$would be suppressed $[97,98]$. This scenario is supported by a recent $2 \mathrm{PP}$ study of slow relaxation of anion states of $\mathrm{C}_{60}$ on graphite [100]. Thus, we predict that the strongly enhanced hot electron generation and slow molecular resonance relaxation are likely to promote efficient hot electronmediated surface femtochemistry on graphitic surfaces.

Finally, our study motivates a mechanism for the femtosecond laser-driven conversion of graphite to nanodiamonds, which has been observed in femtosecond laser excitation at higher fluences than used in our study $[74,75]$. Transforming $\mathrm{sp}^{2}$-to-sp $\mathrm{s}^{3}$ carbon requires weakening of the in-plane conjugation of $\mathrm{C}=\mathrm{C}$ bonds to promote out-ofplane distortion of the molecular sheets and simultaneous introduction of electrons into the interlayer region where they are primed to form interplane $\mathrm{C}-\mathrm{C}$ bonds. This can be accomplished by ultrafast optical heating of the electronic system, such as that achieved in our experiment, which transfers electrons from the occupied $\pi$ bands to the unoccupied $\sigma$ interlayer band. A glimpse of such a phase transition has already been seen in a time domain by ultrafast electron diffraction [74]. Therefore, our study provides a plausible mechanism and physical principles for optimizing the hot electron-driven transmutation of graphite to diamond.

\section{ACKNOWLEDGMENTS}

This research was supported by NSF Grant No. CHE1414466.
[1] M. Breusing, C. Ropers, and T. Elsaesser, Ultrafast Carrier Dynamics in Graphite, Phys. Rev. Lett. 102, 086809 (2009).

[2] Y. Ishida, T. Togashi, K. Yamamoto, M. Tanaka, T. Taniuchi, T. Kiss, M. Nakajima, T. Suemoto, and S. Shin, Non-Thermal Hot Electrons Ultrafastly Generating Hot Optical Phonons in Graphite, Sci. Rep. 1, 64 (2011).

[3] S. Gilbertson, G. L. Dakovski, T. Durakiewicz, J.-X. Zhu, K. M. Dani, A. D. Mohite, A. Dattelbaum, and G. Rodriguez, Tracing Ultrafast Separation and Coalescence of Carrier Distributions in Graphene with Time-Resolved Photoemission, J. Phys. Chem. Lett. 3, 64 (2012).

[4] A. Tomadin, D. Brida, G. Cerullo, A. C. Ferrari, and M. Polini, Nonequilibrium Dynamics of Photoexcited Electrons in Graphene: Collinear Scattering, Auger Processes, and the Impact of Screening, Phys. Rev. B 88, 035430 (2013).

[5] J. C. Johannsen et al., Direct View of Hot Carrier Dynamics in Graphene, Phys. Rev. Lett. 111, 027403 (2013).

[6] I. Gierz, S. Link, U. Starke, and A. Cavalleri, NonEquilibrium Dirac Carrier Dynamics in Graphene Investigated with Time- and Angle-Resolved Photoemission Spectroscopy, Faraday Discuss. 171, 311 (2014).

[7] I. Gierz et al., Tracking Primary Thermalization Events in Graphene with Photoemission at Extreme Time Scales, Phys. Rev. Lett. 115, 086803 (2015).

[8] M. Breusing, S. Kuehn, T. Winzer, E. Malić, F. Milde, N. Severin, J. P. Rabe, C. Ropers, A. Knorr, and T. Elsaesser, Ultrafast Nonequilibrium Carrier Dynamics in a Single Graphene Layer, Phys. Rev. B 83, 153410 (2011).

[9] K. F. Mak, L. Ju, F. Wang, and T.F. Heinz, Optical Spectroscopy of Graphene: From the Far Infrared to the Ultraviolet, Solid State Commun. 152, 1341 (2012).

[10] V. N. Kotov, B. Uchoa, V. M. Pereira, F. Guinea, and A. H. Castro Neto, Electron-Electron Interactions in Graphene: Current Status and Perspectives, Rev. Mod. Phys. 84, 1067 (2012).

[11] F. J. García de Abajo, Graphene Plasmonics: Challenges and Opportunities, ACS Photonics 1, 135 (2014).

[12] H. Zhang, Ultrathin Two-Dimensional Nanomaterials, ACS Nano 9, 9451 (2015).

[13] D. N. Basov, M. M. Fogler, and F. J. García de Abajo, Polaritons in van der Waals Materials, Science 354 (2016).

[14] C. H. Lui, K. F. Mak, J. Shan, and T. F. Heinz, Ultrafast Photoluminescence from Graphene, Phys. Rev. Lett. 105, 127404 (2010).

[15] W.-T. Liu, S. W. Wu, P. J. Schuck, M. Salmeron, Y. R. Shen, and F. Wang, Nonlinear Broadband Photoluminescence of Graphene Induced by Femtosecond Laser Irradiation, Phys. Rev. B 82, 081408 (2010).

[16] T. Li, L. Luo, M. Hupalo, J. Zhang, M. C. Tringides, J. Schmalian, and J. Wang, Femtosecond Population Inversion and Stimulated Emission of Dense Dirac Fermions in Graphene, Phys. Rev. Lett. 108, 167401 (2012).

[17] W. Strek, B. Cichy, L. Radosinski, P. Gluchowski, L. Marciniak, M. Lukaszewicz, and D. Hreniak, LaserInduced White-Light Emission from Graphene CeramicsOpening a Band Gap in Graphene, Light Sci. Appl. 4, e237 (2015). 
[18] T. Winzer, A. Knorr, and E. Malic, Carrier Multiplication in Graphene, Nano Lett. 10, 4839 (2010).

[19] R. Jago, T. Winzer, A. Knorr, and E. Malic, Graphene as Gain Medium for Broadband Lasers, Phys. Rev. B 92, 085407 (2015).

[20] K. J. Tielrooij, M. Massicotte, L. Piatkowski, A. Woessner, Q. Ma, P. Jarillo-Herrero, N. F. v. Hulst, and F. H. L. Koppens, Hot-Carrier Photocurrent Effects at GrapheneMetal Interfaces, J. Phys. Condens. Matter 27, 164207 (2015).

[21] D. P. DiVincenzo and E. J. Mele, Self-Consistent EffectiveMass Theory for Intralayer Screening in Graphite Intercalation Compounds, Phys. Rev. B 29, 1685 (1984).

[22] P. M. Echenique, J. M. Pitarke, E. V. Chulkov, and A. Rubio, Theory of Inelastic Lifetimes of Low-Energy Electrons in Metals, Chem. Phys. 251, 1 (2000).

[23] C. D. Spataru, M. A. Cazalilla, A. Rubio, L. X. Benedict, P. M. Echenique, and S. G. Louie, Anomalous Quasiparticle Lifetime in Graphite: Band Structure Effects, Phys. Rev. Lett. 87, 246405 (2001).

[24] M. Vos, A. S. Kheifets, E. Weigold, and F. Aryasetiawan, Electron Correlation Effects in the Spectral Momentum Density of Graphite, Phys. Rev. B 63, 033108 (2001).

[25] A. G. Marinopoulos, L. Reining, V. Olevano, A. Rubio, T. Pichler, X. Liu, M. Knupfer, and J. Fink, Anisotropy and Interplane Interactions in the Dielectric Response of Graphite, Phys. Rev. Lett. 89, 076402 (2002).

[26] V. Perebeinos and P. Avouris, Impact Excitation by Hot Carriers in Carbon Nanotubes, Phys. Rev. B 74, 121410 (2006).

[27] A. Grüneis et al., Electron-Electron Correlation in Graphite: A Combined Angle-Resolved Photoemission and FirstPrinciples Study, Phys. Rev. Lett. 100, 037601 (2008).

[28] I. Shchatsinin, T. Laarmann, N. Zhavoronkov, C. P. Schulz, and I. V. Hertel, Ultrafast Energy Redistribution in C60 Fullerenes: A Real Time Study by Two-Color Femtosecond Spectroscopy, J. Chem. Phys. 129, 204308 (2008).

[29] A. H. Castro Neto, F. Guinea, N. M. R. Peres, K. S. Novoselov, and A. K. Geim, The Electronic Properties of Graphene, Rev. Mod. Phys. 81, 109 (2009).

[30] L. Brus, Size, Dimensionality, and Strong Electron Correlation in Nanoscience, Acc. Chem. Res. 47, 2951 (2014).

[31] M. Guzzo, J. J. Kas, L. Sponza, C. Giorgetti, F. Sottile, D. Pierucci, M. G. Silly, F. Sirotti, J. J. Rehr, and L. Reining, Multiple Satellites in Materials with Complex Plasmon Spectra: From Graphite to Graphene, Phys. Rev. B 89, 085425 (2014).

[32] P. M. Echenique, R. Berndt, E. V. Chulkov, Th. Fauster, A. Goldmann, and U. Höfer, Decay of Electronic Excitations at Metal Surfaces, Surf. Sci. Rep. 52, 219 (2004).

[33] M. Bauer, A. Marienfeld, and M. Aeschlimann, Hot Electron Lifetimes in Metals Probed by Time-Resolved Two-Photon Photoemission, Prog. Surf. Sci. 90, 319 (2015).

[34] K. Ishioka, M. Hase, M. Kitajima, L. Wirtz, A. Rubio, and H. Petek, Ultrafast Electron-Phonon Decoupling in Graphite, Phys. Rev. B 77, 121402 (2008).

[35] A. Grüneis, C. Attaccalite, L. Wirtz, H. Shiozawa, R. Saito, T. Pichler, and A. Rubio, Tight-Binding Description of the Quasiparticle Dispersion of Graphite and Few-Layer Graphene, Phys. Rev. B 78, 205425 (2008).
[36] P. Cudazzo, I. V. Tokatly, and A. Rubio, Dielectric Screening in Two-Dimensional Insulators: Implications for Excitonic and Impurity States in Graphene, Phys. Rev. B 84, 085406 (2011).

[37] R. Huber, F. Tauser, A. Brodschelm, M. Bichler, G. Abstreiter, and A. Leitenstorfer, How Many-Particle Interactions Develop After Ultrafast Excitation of an Electron-Hole Plasma, Nature (London) 414, 286 (2001).

[38] X. Cui, C. Wang, A. Argondizzo, S. Garrett-Roe, B. Gumhalter, and H. Petek, Transient Excitons at Metal Surfaces, Nat. Phys. 10, 505 (2014).

[39] S. Xu, J. Cao, C. C. Miller, D. A. Mantell, R. J. D. Miller, and Y. Gao, Energy Dependence of Electron Lifetime in Graphite Observed with Femtosecond Photoemission Spectroscopy, Phys. Rev. Lett. 76, 483 (1996).

[40] J. Lehmann, M. Merschdorf, A. Thon, S. Voll, and W. Pfeiffer, Properties and Dynamics of the Image Potential States on Graphite Investigated by Multiphoton Photoemission Spectroscopy, Phys. Rev. B 60, 17037 (1999).

[41] G. Moos, C. Gahl, R. Fasel, M. Wolf, and T. Hertel, Anisotropy of Quasiparticle Lifetimes and the Role of Disorder in Graphite from Ultrafast Time-Resolved Photoemission Spectroscopy, Phys. Rev. Lett. 87, 267402 (2001).

[42] S. Pagliara, M. Montagnese, S. Dal Conte, G. Galimberti, G. Ferrini, and F. Parmigiani, Insight on the Interaction Between Image Potential State and $\pi$ Bands in Graphite, Phys. Rev. B 87, 045427 (2013).

[43] D. Brida et al., Ultrafast Collinear Scattering and Carrier Multiplication in Graphene, Nat. Commun. 4, 1987 (2013).

[44] K. J. Tielrooij, J. C. W. Song, S. A. Jensen, A. Centeno, A. Pesquera, A. Zurutuza Elorza, M. Bonn, L. S. Levitov, and F. H. L. Koppens, Photoexcitation Cascade and Multiple Hot-Carrier Generation in Graphene, Nat. Phys. 9, 248 (2013).

[45] J. C. W. Song and L. S. Levitov, Energy Flows in Graphene: Hot Carrier Dynamics and Cooling, J. Phys. Condens. Matter 27, 164201 (2015).

[46] M. Trushin et al., Ultrafast Pseudospin Dynamics in Graphene, Phys. Rev. B 92, 165429 (2015).

[47] L. Zheng and S. D. Sarma, Comment on "Energy Dependence of Electron Lifetime in Graphite Observed with Femtosecond Photoemission Spectroscopy," Phys. Rev. Lett. 77, 1410 (1996).

[48] S. Xu, J. Cao, C. C. Miller, D. A. Mantell, R. J. D. Miller, and Y. Gao, Reply to the Comment by Lian Zheng and S. Das Sarma, Phys. Rev. Lett. 77, 1411 (1996).

[49] K. Takahashi, J. Azuma, and M. Kamada, TwoDimensional Band Dispersion and Momentum-Resolved Lifetime of the Image-Potential State on Graphite Studied by Angle-Resolved Multiphoton Photoemission Spectroscopy, Phys. Rev. B 85, 075325 (2012).

[50] K. Takahashi, M. Imamura, I. Yamamoto, J. Azuma, and M. Kamada, Image Potential States in Monolayer, Bilayer, and Trilayer Epitaxial Graphene Studied with Time- and Angle-Resolved Two-Photon Photoemission Spectroscopy, Phys. Rev. B 89, 155303 (2014).

[51] D. Gugel, D. Niesner, C. Eickhoff, S. Wagner, M. Weinelt, and Th. Fauster, Two-Photon Photoemission from ImagePotential States of Epitaxial Graphene, 2D Mater. 2, 045001 (2015). 
[52] Th. Fauster, F. J. Himpsel, J. E. Fischer, and E. W. Plummer, Three-Dimensional Energy Band in Graphite and Lithium-Intercalated Graphite, Phys. Rev. Lett. 51, 430 (1983).

[53] D. A. Fischer, R. M. Wentzcovitch, R. G. Carr, A. Continenza, and A.J. Freeman, Graphitic Interlayer States: A Carbon K Near-Edge X-Ray-Absorption FineStructure Study, Phys. Rev. B 44, 1427 (1991).

[54] M. Posternak, A. Baldereschi, A. J. Freeman, and E. Wimmer, Prediction of Electronic Surface States in Layered Materials: Graphite, Phys. Rev. Lett. 52, 863 (1984).

[55] T. Kihlgren, T. Balasubramanian, L. Wallden, and R. Yakimova, K/graphite: Uniform Energy Shifts of Graphite Valence States, Surf. Sci. 600, 1160 (2006).

[56] V. M. Silkin, J. Zhao, F. Guinea, E. V. Chulkov, P. M. Echenique, and H. Petek, Image Potential States in Graphene, Phys. Rev. B 80, 121408 (2009).

[57] M. Feng, J. Zhao, T. Huang, X.-Y. Zhu, and H. Petek, The Electronic Properties of Superatom States of Hollow Molecules, Acc. Chem. Res. 44, 360 (2011).

[58] L. Yang, J. Deslippe, C.-H. Park, M. L. Cohen, and S. G. Louie, Excitonic Effects on the Optical Response of Graphene and Bilayer Graphene, Phys. Rev. Lett. 103, 186802 (2009).

[59] R. Saito, A. Grüneis, G. G. Samsonidze, G. Dresselhaus, M. S. Dresselhaus, A. Jorio, L. G. Cançado, M. A. Pimenta, and A. G. Souza Filho, Optical Absorption of Graphite and Single-Wall Carbon Nanotubes, Appl. Phys. A 78, 1099 (2004).

[60] E. Malic, T. Winzer, E. Bobkin, and A. Knorr, Microscopic Theory of Absorption and Ultrafast Many-Particle Kinetics in Graphene, Phys. Rev. B 84, 205406 (2011).

[61] E. Malic, T. Winzer, and A. Knorr, Efficient Orientational Carrier Relaxation in Optically Excited Graphene, Appl. Phys. Lett. 101, 213110 (2012).

[62] X.-Q. Yan, J. Yao, Z.-B. Liu, X. Zhao, X.-D. Chen, C. Gao, W. Xin, Y. Chen, and J.-G. Tian, Evolution of Anisotropic-to-Isotropic Photoexcited Carrier Distribution in Graphene, Phys. Rev. B 90, 134308 (2014).

[63] J. J. Quinn, Range of Excited Electrons in Metals, Phys. Rev. 126, 1453 (1962).

[64] V.A. Gasparov and R. Huguenin, Electron-Phonon, Electron-Electron and Electron-Surface Scattering in Metals from Ballistic Effects, Adv. Phys. 42, 393 (1993).

[65] E. H. Hwang, B. Y.-K. Hu, and S. Das Sarma, Inelastic Carrier Lifetime in Graphene, Phys. Rev. B 76, 115434 (2007).

[66] M. Achermann, A. P. Bartko, J. A. Hollingsworth, and V. I. Klimov, The Effect of Auger Heating on Intraband Carrier Relaxation in Semiconductor Quantum Rods, Nat. Phys. 2, 557 (2006).

[67] X. Y. Wang, D. M. Riffe, Y. S. Lee, and M. C. Downer, Time-Resolved Electron-Temperature Measurement in a Highly Excited Gold Target Using Femtosecond Thermionic Emission, Phys. Rev. B 50, 8016 (1994).

[68] F. Bisio, M. Nývlt, J. Franta, H. Petek, and J. Kirschner, Mechanisms of High-Order Perturbative Photoemission from $\mathrm{Cu}(001)$, Phys. Rev. Lett. 96, 087601 (2006).

[69] A. Winkelmann, W.-C. Lin, C.-T. Chiang, F. Bisio, H. Petek, and J. Kirschner, Resonant Coherent Three-Photon
Photoemission from $\mathrm{Cu}(001)$, Phys. Rev. B 80, 155128 (2009).

[70] M. Krüger, M. Schenk, M. Förster, and P. Hommelhoff, Attosecond Physics in Photoemission from a Metal Nanotip, J. Phys. B 45, 074006 (2012).

[71] T. Ohta, A. Bostwick, J. L. McChesney, T. Seyller, K. Horn, and E. Rotenberg, Interlayer Interaction and Electronic Screening in Multilayer Graphene Investigated with Angle-Resolved Photoemission Spectroscopy, Phys. Rev. Lett. 98, 206802 (2007).

[72] From the perspective of this article, electron-electron scattering processes and screening of the Coulomb interaction in graphene and graphite are similar. The main difference between the materials is that multiple graphene sheets are necessary for the interlayer band to exist.

[73] M. Bonn, S. Funk, C. Hess, D. N. Denzler, C. Stampfl, M. Scheffler, M. Wolf, and G. Ertl, Phonon-Versus ElectronMediated Desorption and Oxidation of $\mathrm{CO}$ on $\mathrm{Ru}(0001)$, Science 285, 1042 (1999).

[74] R. K. Raman, Y. Murooka, C.-Y. Ruan, T. Yang, S. Berber, and D. Tománek, Direct Observation of Optically Induced Transient Structures in Graphite Using Ultrafast Electron Crystallography, Phys. Rev. Lett. 101, 077401 (2008).

[75] J. Kanasaki, E. Inami, K. Tanimura, H. Ohnishi, and K. Nasu, Formation of $\mathrm{sp}^{3}$-Bonded Carbon Nanostructures by Femtosecond Laser Excitation of Graphite, Phys. Rev. Lett. 102, 087402 (2009).

[76] D. Wegkamp and J. Stähler, Ultrafast Dynamics During the Photoinduced Phase Transition in $\mathrm{VO}_{2}$, Prog. Surf. Sci. 90, 464 (2015).

[77] J. Narayan and A. Bhaumik, Novel Phase of Carbon, Ferromagnetism, and Conversion into Diamond, J. Appl. Phys. 118, 215303 (2015).

[78] A. Argondizzo, X. Cui, C. Wang, H. Sun, H. Shang, J. Zhao, and H. Petek, Ultrafast Multiphoton Pump-Probe Photoemission Excitation Pathways in Rutile $\mathrm{TiO}_{2}$, Phys. Rev. B 91, 155429 (2015).

[79] S. Ogawa, H. Nagano, H. Petek, and A. P. Heberle, Optical Dephasing in Cu(111) Measured by Interferometric TwoPhoton Time-Resolved Photoemission, Phys. Rev. Lett. 78, 1339 (1997).

[80] H. Petek, A. P. Heberle, W. Nessler, H. Nagano, S. Kubota, S. Matsunami, N. Moriya, and S. Ogawa, Optical Phase Control of Coherent Electron Dynamics in Metals, Phys. Rev. Lett. 79, 4649 (1997).

[81] H. Petek and S. Ogawa, Femtosecond Time-Resolved Two-Photon Photoemission Studies of Electron Dynamics in Metals, Prog. Surf. Sci. 56, 239 (1997).

[82] Th. Fauster, C. Reuß, I. L. Shumay, and M. Weinelt, Femtosecond Two-Photon Photoemission Studies of Image-Potential States, Chem. Phys. 251, 111 (2000).

[83] A. Winkelmann, C.-T. Chiang, F. Bisio, W.-C. Lin, J. Kirschner, and H. Petek, in Dynamics at Solid State Surfaces and Interfaces, edited by U. Bovensiepen, $\mathrm{H}$. Petek, and M. Wolf (Wiley-VCH Verlag, Weinheim, 2010).

[84] S. W. Jung, W. J. Shin, J. Kim, L. Moreschini, H. W. Yeom, E. Rotenberg, A. Bostwick, and K.S. Kim, Sublattice Interference as the Origin of $\sigma$ Band Kinks in Graphene, Phys. Rev. Lett. 116, 186802 (2016). 
[85] S. Y. Zhou, G. H. Gweon, and A. Lanzara, Low Energy Excitations in Graphite: The Role of Dimensionality and Lattice Defects, Ann. Phys. (Amsterdam) 321, 1730 (2006).

[86] K. Hansen, K. Hoffmann, and E. E. B. Campbell, Thermal Electron Emission from the Hot Electronic Subsystem of Vibrationally Cold $\mathrm{C}_{60}$, J. Chem. Phys. 119, 2513 (2003).

[87] M. Vahdani Moghaddam, P. Yaghoobi, G. A. Sawatzky, and A. Nojeh, Photon-Impenetrable, Electron-Permeable: The Carbon Nanotube Forest as a Medium for Multiphoton Thermal-Photoemission, ACS Nano 9, 4064 (2015).

[88] M. J. Weida, S. Ogawa, H. Nagano, and H. Petek, Ultrafast Interferometric Pump-Probe Correlation Measurements in Systems with Broadened Bands or Continua, J. Opt. Soc. Am. B 17, 1443 (2000).

[89] R. K. Raman, Z. Tao, T.-R. Han, and C.-Y. Ruan, Ultrafast Imaging of Photoelectron Packets Generated from Graphite Surface, Appl. Phys. Lett. 95, 181108 (2009).

[90] S. Pagliara, G. Galimberti, S. Mor, M. Montagnese, G. Ferrini, M. S. Grandi, P. Galinetto, and F. Parmigiani, Photoinduced $\pi-\pi^{*}$ Band Gap Renormalization in Graphite, J. Am. Chem. Soc. 133, 6318 (2011).

[91] J. Lehmann, M. Merschdorf, W. Pfeiffer, A. Thon, S. Voll, and G. Gerber, Surface Plasmon Dynamics in Silver Nanoparticles Studied by Femtosecond Time-Resolved Photoemission, Phys. Rev. Lett. 85, 2921 (2000).

[92] A. Kubo, K. Onda, H. Petek, Z. Sun, Y. S. Jung, and H. K. Kim, Femtosecond Imaging of Surface Plasmon Dynamics in a Nanostructured Silver Film, Nano Lett. 5, 1123 (2005).

[93] M. Aeschlimann et al., Perfect Absorption in Nanotextured Thin Films via Anderson-Localized Photon Modes, Nat. Photonics 9, 663 (2015).

[94] H. Petek, H. Nagano, M. J. Weida, and S. Ogawa, The Role of Auger Decay in Hot Electron Excitation in Copper, Chem. Phys. 251, 71 (2000).

[95] M. Lisowski, P. A. Loukakos, U. Bovensiepen, J. Stähler, C. Gahl, and M. Wolf, Ultra-Fast Dynamics of Electron Thermalization, Cooling and Transport Effects in Ru(001), Appl. Phys. A 78, 165 (2004).

[96] R. R. Cavanagh, D. S. King, J. C. Stephenson, and T. F. Heinz, Dynamics of Nonthermal Reactions: Femtosecond Surface Chemistry, J. Phys. Chem. 97, 786 (1993).

[97] J. T. Yates, Jr. and H. Petek, Introduction: Photochemistry and Photophysics on Surfaces, Chem. Rev. 106, 4113 (2006).

[98] R. D. Muiño, D. Sánchez-Portal, V. M. Silkin, E. V. Chulkov, and P. M. Echenique, Time-Dependent Electron Phenomena at Surfaces, Proc. Natl. Acad. Sci. U.S.A. 108, 971 (2011).

[99] H. Petek, Photoexcitation of Adsorbates on Metal Surfaces: One-Step or Three-Step, J. Chem. Phys. 137, 091704 (2012).

[100] M. Shibuta, K. Yamamoto, T. Ohta, M. Nakaya, T. Eguchi, and A. Nakajima, Direct Observation of Photocarrier Electron Dynamics in $\mathrm{C}_{60}$ Films on Graphite by TimeResolved Two-Photon Photoemission, Sci. Rep. 6, 35853 (2016). 